Meeting abstract

\title{
Flipping NextGen: using biological systems to characterize NextGen sequencing technologies
}

\author{
Jarret Glasscock*, Ryan Richt and Matt Hickenbotham
}

Address: Cofactor Genomics, St. Louis, MO 63103, USA

Email: Jarret Glasscock* - Jarret_Glasscock@cofactorgenomics.com

* Corresponding author

from UT-ORNL-KBRIN Bioinformatics Summit 2009

Pikeville, TN, USA. 20-22 March 2009

Published: 25 June 2009

BMC Bioinformatics 2009, 10(Suppl 7):AI8 doi:10.1 I86/I47I-2105-I0-S7-AI8

This abstract is available from: http://www.biomedcentral.com/I47I-2I05/I0/S7/AI8

(c) 2009 Glasscock et al; licensee BioMed Central Ltd.

\section{Background}

At a current 12 gigabases per sequencing run (and growing), there have been significant advancements in DNA sequencing technologies resulting in next generation (NextGen) sequencing platforms that produce 5 orders of magnitude more data than platforms used for the human genome project.

\section{Results}

A broad range of genomes was surveyed in order to assess characteristics necessary to sufficiently analyze these biological systems. In the context of genome re-sequencing projects we found 15 bp was needed to uniquely map $98 \%$ of loci in many bacteria, while 20 bp was needed before hitting lower asymptotes to uniquely characterize a fraction of more complex genomes (Figure 1).

Transcriptomes on the other hand were much less variable and required fewer bases $(x)$ to uniquely map a much larger percentage (y) of their sequence space. For example, more than $98 \%$ of the complex human transcriptome could be uniquely characterized with as few as $20 \mathrm{bp}$.

Finally, de-novo sequencing (i.e. without a reference) would require a minimum of $1 / 2$ of the sequence length to be unique in order to allow sufficient contig extension in the assembly process. For example, 40-50 bp reads are necessary for de-novo characterization of these systems uniquely defined by 20-25 bp reads. As of 2009, short read NextGen sequencing technologies have moved to 50 bp and beyond, ushering in what is expected to be the start of a revolution in genomics.

\section{Conclusion}

These results establish a lower bound on sequence length (x) required to sufficiently conduct re-sequencing, transcriptome, and de-novo sequencing projects. The asymptotic nature of the results also provides a guide for what percentage of the total space (y) we might expect to define in genomes/transcriptomes of similar size and complexity.

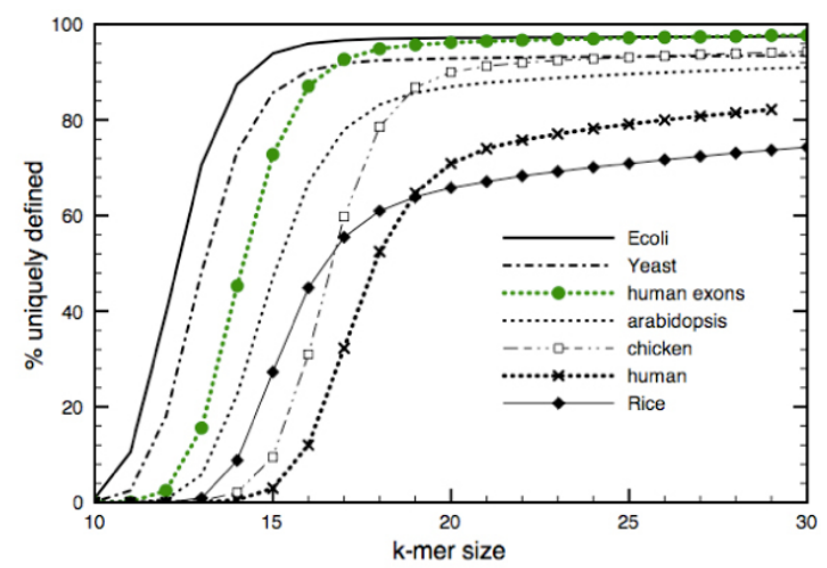

Figure I

Percent of genome or transcriptome ( $Y$-axis) uniquely defined by a read length ( $X$-axis). 